\title{
Effect of Conductivity on Magneto-Gravitational Instability and Suspended Particles
}

\author{
R. K. Chhajlani, P. Vashistha*, and S. C. Bhand \\ School of Studies in Physics, Vikram University, Ujjain, M.P., India \\ Z. Naturforsch. 33a, 1469-1471 (1978); received November 3, 1978 \\ The self gravitational instability of an infinite homogeneous magnetized gas-particle medium \\ in the presence of suspended particles is investigated. The conductivity of the medium is assumed \\ to be finite. The dispersion relation is obtained for such a medium. It is found that in the presence \\ of suspended particles a sufficient condition of instability is obtained by Jeans' criterion for a \\ self gravitating magnetized conducting gas-particle medium.
}

\section{Introduction}

The problem of self gravitational instability of an infinite homogeneous medium was first considered by Jeans [1]. A comprehensive account of the investigations of this problem under different conditions has been given by Chandrasekhar [2]. $\mathrm{He}$ has found that Jeans' criterion remains unaffected by the presence of a uniform magnetic field.

Sharma et al. [3] have carried out an investigation on the effect of suspended particles on the onset of Benard convection in hydromagnetics. They have found that the effect of suspended particles is to destabilize the layer and that the magnetic field has a stabilizing influence. Further, Sharma [4] has considered the effect of suspended particles on the gravitational instability of an infinite homogeneous gas-particle medium. He [5] also investigated the effect of a magnetic field on the gravitational instability of an infinite conducting homogeneous self gravitating gas-particle medium in the presence of suspended particles.

The object of the present investigation is to study the gravitational instability of an infinite homogeneous magnetized self gravitating gasparticle medium. The conductivity of the medium is assumed to be finite and its effect on the stability of the medium is discussed. Suspended or dust particles are present in the interstellar gas and the effect of finite conductivity on the gravitational instability of an interstellar medium is also important. In view of the importance of this problem, a detailed investigation is carried out.

* Madhav Science College, Vikram University, Ujjain M.P., India.

Reprint requests to Dr. R. K. Chhajlani, Near Kanchan Bhawan, Var Ruchi Marg, Madhav Nagar, Ujjain (M.P.), Indien.

\section{Perturbed Equations}

The linearized perturbation equations for a self gravitating magnetized and finite conducting medium in the presence of suspended particles are

$$
\begin{aligned}
\frac{\partial \boldsymbol{u}}{\partial t}= & -\frac{1}{\varrho} \nabla \delta p+\nabla \delta U+\frac{k N}{\varrho}(\boldsymbol{v}-\boldsymbol{u}) \\
& +v\left[\nabla^{2} \boldsymbol{u}+\frac{1}{3} \nabla(\nabla \cdot \boldsymbol{u})\right] \\
& +\frac{1}{4 \pi \varrho}(\nabla \times \boldsymbol{h}) \times \boldsymbol{H}, \\
\partial(\delta \varrho) / \partial t & =-\varrho \nabla \cdot \boldsymbol{u}, \\
\delta p & =c^{2} \delta \varrho, \\
\nabla \cdot \boldsymbol{h} & =0 \\
(\tau \partial / \partial t & +1) \boldsymbol{v}=\boldsymbol{u}, \\
\partial \boldsymbol{h} / \partial t & =\nabla \times(\boldsymbol{u} \times \boldsymbol{H})+\eta \nabla^{2} \boldsymbol{h}, \\
\nabla^{2} \delta U & =-4 \pi G \delta \varrho,
\end{aligned}
$$

where $\boldsymbol{u}(u, v, w), \delta \varrho, \delta p, \delta U$ denote the perturbations in gas velocity, density $\varrho$, pressure $p$ and self gravitational potential $U$, respectively. $N(\boldsymbol{x}, t)$ and $v(\boldsymbol{x}, t)$ denote the number density and velocity field of the particles, $k=6 \pi \varrho v r$ is a constant, $r$ is the particle radius, $v$ is the kinematic viscosity of the gas $\boldsymbol{x}=(x, y, z), c$ denotes the velocity of sound in the medium and $G$ denotes the gravitational constant. $\boldsymbol{h}\left(h_{x}, h_{y}, h_{z}\right)$ is the perturbation in the magnetic field $\boldsymbol{H}(0,0, H) \cdot \eta$ is the conductivity. $\tau=m / k$, and $m N$ is the mass of the particles per unit volume.

In writing the equation of motion for the particles, we have neglected the buoyancy force as its stabilizing effect for the case of two free boundaries is extremely small. Interparticle reactions are ignored (Scanlon and Segel [6]). In writing (1), use has been made of Stokes' assumption that the bulk viscosity $\lambda+\frac{2}{3} \varrho v=0$. 


\section{Dispersion Relation and Discussion}

We assume that all the perturbed quantities vary in the form $\exp i\left(k_{x} x+k_{z} z+\sigma t\right)$, where $\sigma$ is the growth rate of perturbation and $k_{x}$ and $k_{z}$ are the wave numbers of the perturbation along the $x$ and $z$ axes.

We get the following equations from (1), using $(2)-(7)$ and variation:

$$
\begin{aligned}
& {\left[\left\{-\sigma^{2} \tau+i \sigma\left(1+\frac{k N \tau}{\varrho}+\frac{4}{3} \nu k^{2} \tau\right)+\frac{4}{3} \nu k^{2}\right\}\left(i \sigma+\eta k^{2}\right)+V^{2} k^{2}(\tau i \sigma+1)\right] u} \\
& =-\frac{\Omega_{j}^{2}}{k^{2}}(1+\tau i \sigma)\left(i \sigma+\eta k^{2}\right) i k_{x} s, \\
& {\left[V^{2}(\tau i \sigma+1) k_{z}^{2}+\left(i \sigma+\eta k^{2}\right)\left\{-\sigma^{2} \tau+i \sigma\left(1+\frac{k N \tau}{\varrho}+\frac{4}{3} v k^{2} \tau\right)+\frac{4}{3} \nu k^{2}\right\}\right] v=0,} \\
& {\left[-\sigma^{2} \tau+i \sigma\left(1+\frac{k N \tau}{\varrho}+\frac{4}{3} v k^{2} \tau\right)+\frac{4}{3} v k^{2}\right] w=-\frac{\Omega_{j}^{2}}{k^{2}}(1+\tau i \sigma) i k_{z} s .}
\end{aligned}
$$

On taking the divergence of (1), using $(2)-(7)$ and performing the above said variation we get

$$
\begin{aligned}
{\left[i \sigma^{3} \tau\right.} & \left.+\sigma^{2}\left(1+\frac{k N \tau}{\varrho} \tau+\frac{4}{3} v k^{2} \tau\right)-\frac{4}{3} v k^{2} i \sigma-\Omega_{j}^{2}(1+\tau i \sigma)\right]\left(i \sigma+\eta k^{2}\right) s \\
& +i k_{x} V^{2} k^{2}(\tau i \sigma+1) u=0 .
\end{aligned}
$$

(8)-(11) can be written in the form

$$
\left|\begin{array}{cccc}
p \alpha+V^{2} k^{2} \beta & 0 & 0 & \frac{\Omega_{j}^{2}}{k^{2}} \alpha i k_{x} \beta \\
0 & p \alpha+V^{2} k_{z}^{2} \beta & 0 & 0 \\
0 & 0 & p & \frac{\Omega_{j}^{2}}{k^{2}} \beta i k_{z} \\
i k_{x} V^{2} k^{2} \beta & 0 & 0 & \alpha\left[-i \sigma p-\Omega_{j}^{2} \beta\right]
\end{array}\right|\left|\begin{array}{l}
u \\
v \\
w \\
s
\end{array}\right|=0,
$$

where

$$
\begin{aligned}
V^{2} & =H^{2} / 4 \pi \varrho, \quad \Omega_{j}^{2}=c^{2} k^{2}-4 \pi G \varrho, \\
s & =\delta \varrho / \varrho, \quad x=\left(1+\frac{4}{3} v k^{2} \tau+\frac{k N \tau}{\varrho}\right), \\
p & =-\tau \sigma^{2}+i \sigma\left(1+\frac{4}{3} \nu k^{2} \tau+\frac{k N \tau}{\varrho}\right)+\frac{4}{3} \nu k^{2}, \\
\alpha & =i \sigma+\eta k^{2}, \quad \beta=(1+\tau i \sigma), \\
k^{2} & =k_{x}{ }^{2}+k_{z}^{2} .
\end{aligned}
$$

The determinant of the matrix of (12) gives the dispersion relation $\left[i \sigma+\eta k^{2}\right]\left[p\left(i \sigma+\eta k^{2}\right)+V^{2} k_{z}^{2}(\tau i \sigma+1)\right][p]$

$\cdot\left[\left\{p\left(i \sigma+\eta k^{2}\right)+V^{2} k^{2}(\tau i \sigma+1)\right\}\left\{-i \sigma p-\Omega_{j}^{2}(1+\tau i \sigma)\right\}-\frac{\Omega_{j}^{2}}{k^{2}}(1+\tau i \sigma)^{2} k_{x^{2}} V^{2} k^{2}\right]=0$.

The first factor of the dispersion relation gives

$$
\sigma=i \eta k^{2} \text {. }
$$

(14) gives a viscous type of damped mode modified by the finite conductivity, which is stable.

The second factor gives

$$
\begin{aligned}
\omega^{3} \tau & +\omega^{2}\left[\left(1+\frac{k N \tau}{\varrho}+\frac{4}{3} v k^{2} \tau\right)+\eta k^{2} \tau\right]+\omega\left[\frac{4}{3} v k^{2}+V^{2} k_{z}^{2} \tau+\left(1+\frac{k N \tau}{\varrho}+\frac{4}{3} v k^{2} \tau\right) \eta k^{2}\right] \\
& +V^{2} k_{z^{2}}+{ }_{3}^{4} \nu k^{2} \eta k^{2}=0,
\end{aligned}
$$


where we have put $\omega=i \sigma$. (15) has no positive real root or complex root whose real part is positive. In this respect the system is stable.

Next we get the dispersion relation

$\tau \omega^{2}+\left(1+\frac{4}{3} v k^{2} \tau+\frac{k N \tau}{\varrho}\right) \omega+{ }_{3}^{4} \nu k^{2}=0$.

(16) does not admit a positive real root or complex root whose real part is positive, so the system is stable.

The last factor of (13) gives

$$
\begin{aligned}
& \omega^{6} \tau^{2}+\omega^{5}\left[2 \tau x+\eta k^{2} \tau^{2}\right] \\
& +\omega^{4}\left[x^{2}+\tau\left(\tau \Omega_{j}^{2}+\tau V^{2} k^{2}+\frac{8}{3} \nu k^{2}\right)\right. \\
& \left.+2 \tau x \eta k^{2}\right] \\
& +\omega^{3}\left[x\left(\frac{8}{3} v k^{2}+\tau \Omega_{j}^{2}+\tau V^{2} k^{2}\right)\right. \\
& +\tau\left(\Omega_{j}^{2}+V^{2} k^{2}\right) \\
& \left.+\left(\frac{8}{3} \tau \nu k^{2}+x^{2}+\Omega_{j}^{2} \tau^{2}\right) \eta k^{2}\right] \\
& +\omega^{2}\left[\frac{4}{3} v k^{2}\left(\frac{4}{3} v k^{2}+\Omega_{j}^{2} \tau\right)+\frac{4}{3} \tau k^{4} v V^{2}\right. \\
& +\tau^{2} \Omega_{j}{ }^{2} V^{2} k_{z}{ }^{2}+x\left(V^{2} k^{2}+\Omega_{j}^{2}\right) \\
& \left.+\eta k^{2}\left(\tau \Omega_{j}^{2}+\tau x \Omega_{j}^{2}+\frac{8}{3} x \nu k^{2}\right)\right] \\
& +\omega\left[\Omega_{j}{ }^{2}\left(\frac{4}{3} \nu k^{2}+2 \tau V^{2} k_{z}{ }^{2}\right)+\frac{4}{3} V^{2} k^{4} v\right] \\
& +\eta k^{2}\left\{\left({ }_{3}^{4} v k\right)^{2}+\Omega_{j}^{2} x+{ }_{3}^{4} v k^{2} \Omega_{j}{ }^{2} \tau\right\} \\
& +\left({ }_{3}^{4} v k^{2} \eta k^{2}+V^{2} k_{z}^{2}\right) \Omega_{j}^{2}=0 \text {. }
\end{aligned}
$$

We conclude from (17) that when $\Omega_{j}^{2}<0$, (17) has at least one positive root. This means that at least one value of $i \sigma$ is positive and this gives instability.

Thus, from (17) we note that the condition of instability of the system is

$$
c^{2} k^{2}-4 \pi G \varrho<0 .
$$

This is called Jeans' criterion of instability.

We find that the dispersion relation (13) gives four relations viz. (14), (15), (16) and (17). The first three relations give stable modes in the system whereas Eq. (17) gives Jeans' instability in the medium. Thus we conclude that Jeans' criterion is a sufficient condition for the instability of an infinite, homogeneous magnetized self gravitating gas-particle medium in the presence of suspended particles and finite conductivity of the medium.

\section{Acknowledgements}

The authors are highly grateful to Dr. P.N. Kawthekar, Vice Chancellor of Vikram University, Ujjain, and Dr. H. N. Sharma, Principal, Madhav Science College, Ujjain, for their kind interest in the work.

[4] R. C. Sharma, Z. Appl. Math. Mech. 55, 615 (1975).

[5] R. C. Sharma, Astrophys. Space Sci. 46, 255 (1977).

[6] J. W. Scanlon and L. A. Segel, Phys. Fluids 16, 1573 (1973).
[2] S. Chandrasekhar, Hydrodynamic and Hydromagnetic Stability, Clarendon Press, Oxford 1961.

[3] R. C. Sharma, K. Prakash, and S. N. Dube, Acta Physica Hungarica 40, 2 (1976). 